\title{
Atomistic Simulations of Electrowetting in Carbon Nanotubes
}

\author{
A. Kutana and K. P. Giapis* \\ Division of Chemistry and Chemical Engineering, California Institute of Technology, \\ Pasadena, California 91125
}

Received December 4, 2005; Revised Manuscript Received January 16, 2006

\begin{abstract}
Electrowetting of carbon nanotubes by mercury was studied using classical molecular dynamics simulations in conjunction with a macroscopic electrocapillarity model. A scaled ab initio mercury dimer potential, optimized to reproduce the mercury liquid density (at $300 \mathrm{~K}$ ), melting point, and wetting angle on graphite, was selected for the simulations. Wetting of $(20,20)$ single-walled carbon nanotubes by mercury occurs above a threshold voltage of $2.5 \mathrm{~V}$ applied across the interface. Both the electrocapillary pressure and imbibition velocity increase quadratically with voltage and can acquire large values, for example, $2.4 \mathrm{kbar}$ and $28 \mathrm{~m} / \mathrm{s}$ at $4 \mathrm{~V}$, indicating a notable driving force. The observed voltage scaling can be captured by the Lucas-Washburn equation modified to include a wetting-line friction term.
\end{abstract}

I. Introduction. The long inner core of carbon nanotubes has received attention as a channel for studies of nanoscale capillarity ${ }^{1}$ and as a mold for the formation of continuous nanowires with new electrical and magnetic properties. ${ }^{2-8}$ Such applications require improvements in understanding of the wetting behavior of nanotubes by liquids. Just like macroscopic capillaries, carbon nanotubes can be filled with liquids by capillary forces ${ }^{2,4}$ provided that the surface tension of the liquid is sufficiently low to permit internal wetting. ${ }^{9}$ Liquids with surface tension larger than $180 \mathrm{mN} / \mathrm{m}$ do not wet carbon nanotubes spontaneously. ${ }^{9}$ This condition restricts the choice of liquids, which can enter or be transported through the inner core of a nanotube; in fact, it excludes most metals. In order for a liquid with high surface tension to enter the core, either a large external pressure must be applied $^{6}$ or the surface tension must be reduced. ${ }^{7}$ The latter can be accomplished by the application of an electrical potential across a liquid-solid interface, a phenomenon known as electrocapillarity. ${ }^{10,11}$ Electrically activated wetting and filling of the inner core of individual single-walled nanotubes (SWNTs) by mercury has been recently demonstrated experimentally. ${ }^{12}$ The nanotubes were attached to gold-coated atomic force microscope (AFM) tips, and their suspended open ends were partially immersed into a mercury drop. Application of a rather small voltage $(1-2 \mathrm{~V}$, independent of polarity) to the immersed nanotube resulted in substantially increased conductance, which was attributed to the following: (1) the improved contacts due to better wetting and (2) the filling of the nanotube with mercury and the formation of a metallic nanowire. ${ }^{12} \mathrm{~A}$ remarkable effect

\footnotetext{
* Corresponding author. E-mail: giapis@cheme.caltech.edu.
}

of electrowetting was the transport of mercury through and around the nanotube in a sufficient amount to cause dissolution of the gold from the tip by forming an amalgam. ${ }^{12}$ The time and length scales of the nanotube wetting process make it extremely difficult to observe experimentally the fluid motion inside the core or to measure important parameters such as wetting speed and capillary pressure. However, these scales are amenable to molecular dynamics simulations, which can reveal the wetting dynamics of nanoscale capillarity in full atomistic detail. ${ }^{1}$

We present here a computational study of electrowetting in open SWNTs by mercury using classical molecular dynamics (MD) simulations. Elements of this study have been used to corroborate and explain the experimental results of ref 12 and, in particular, to understand why it is extremely difficult to find mercury in the nanotube core after its extraction from the mercury drop and to establish whether the rate of mercury transport through the nanotube suffices to dissolve the gold-coated AFM tip. In this paper, we present the thermodynamic underpinnings of the electrowetting model used in conjunction with atomistic simulations to reveal the electrowetting dynamics. Particular emphasis is placed on identifying scaling laws for the nanotube filling process, which may be of broader importance for the new field of nanofluidics. We further explore the applicability of macroscopic capillary hydrodynamics to the case of nanotube wetting.

II. Choice of Potentials. The carbon nanotube is assumed to be rigid. Although good potentials for $\mathrm{C}-\mathrm{C}$ interactions are available, they are omitted from the present study for computational efficiency. Accounting for such interactions 
Table 1. Parameters for the Scaled ab Initio Schwerdtfeger Mercury Potential, Described by Equation $1^{a}$

\begin{tabular}{lr}
\hline & \multicolumn{1}{c}{$U_{s}^{*}(r)$} \\
\hline$a^{*}{ }_{6}$ & $-1.036542 \mathrm{e}+02$ \\
$a^{*}$ & $-1.539877 \mathrm{e}+03$ \\
$a^{*}{ }_{10}$ & $4.271609 \mathrm{e}+04$ \\
$a^{*}{ }_{12}$ & $-2.975002 \mathrm{e}+05$ \\
$a^{*}{ }_{14}$ & $9.965436 \mathrm{e}+05$ \\
$a^{*}{ }_{16}$ & $-1.633356 \mathrm{e}+06$ \\
$a^{*}{ }_{18}$ & $1.049907 \mathrm{e}+06$
\end{tabular}
${ }^{a}$ The units of $U_{S}^{*}(r)$ are electronvolts, and the units of distance $r$ are
Angstroms.

in test runs did not materially alter the obtained results while it increased dramatically the computational time required to reach equilibrium for both nonwetting and wetting states.

II.1. $\mathrm{Hg}-\mathrm{Hg}$ Potential. Bonding in mercury is represented by complex interactions where electronic correlations and relativistic effects play an important role. ${ }^{13}$ Initially bound by van der Waals-type forces, mercury clusters become metallic as their size increases from 20 to 70 atoms. ${ }^{14}$ Bulk mercury has a melting temperature of $234 \mathrm{~K}$, unusually low for a metal, below which it assumes a rhombohedral structure. Thus, one might expect that pairwise potentials used for simple liquids would fail to capture the physical properties of the bulk liquid state of such a complex system, and a many-body potential would be required at a minimum. Indeed, we found this to be true for Lennard-Jones (LJ)type potentials, which we deemed inadequate for studies of electrowetting. However, we have shown elsewhere ${ }^{15}$ that an appropriately scaled pairwise potential, based on the ab initio calculations of Schwerdtfeger et al. ${ }^{16}$ for a mercury dimer, can reproduce some of the basic properties of mercury quite well, such as melting point and liquid density at 300 K. This "scaled Schwerdtfeger potential" has the form

$$
U_{S}^{*}(r)=U_{S}(\lambda r)=\sum_{j=3}^{9} a_{2 j}^{*} r^{-2 j}
$$

where $U_{s}(r)$ is the original Schwerdtfeger potential for a mercury dimer, ${ }^{16} \lambda=1.167$ has been introduced to match the density of liquid $\mathrm{Hg}$ at $300 \mathrm{~K}$, and the parameters $a_{2 j}^{*}$ are listed in Table 1.

II.2. $\mathrm{Hg}-\mathrm{C}$ Potential. The mercury-carbon nanotube wall interaction was modeled through a pairwise LJ potential of the form

$$
U_{\mathrm{LJ}}(r)=4 \epsilon\left((\sigma / r)^{12}-(\sigma / r)^{6}\right)
$$

where $r$ is the distance between a pair of mercury atoms. The length scale $\sigma_{\mathrm{HgC}}=3.321 \AA$ was found by applying Lorentz-Berthelot mixing rules to the pair $\sigma_{\mathrm{HgHg}}=3.234$ $\AA$ for the mercury dimer, and $\sigma_{\mathrm{CC}}=3.407 \AA$ for graphite. The second parameter, $\epsilon_{\mathrm{HgC}}$, was obtained from simulating the wetting of a graphite surface by a mercury drop described by the scaled Schwerdtfeger potential. Because the contact angle of the drop depends on the strength of the attraction between individual atoms in the solid and liquid, the pair energy can be calculated from the experimental contact angle of $\theta=152.5^{\circ} .{ }^{17}$ This value is matched in MD simulations ${ }^{15}$ when $\epsilon_{\mathrm{HgC}} / k_{\mathrm{B}}=14.7 \mathrm{~K}$, which is adopted for the present simulations of electrowetting in carbon nanotubes by mercury.

III. Electrowetting Model. According to the macroscopic theory of electrocapillarity, the reduction in surface tension and the ensuing wetting is attributed to the mutual repulsion of charged surface elements acting against surface tension. Originally developed by Lippmann for salt electrolytes in contact with a metal electrode,$^{18}$ the theory is adopted here for a mercury-nanotube contact. A potential is applied at one end of the nanotube, while the other end forms a contact with mercury fluid that is grounded. In order for electrowetting to occur, electric charge must be accumulated across the contact upon application of a voltage. The exact nature of the barrier at the interface responsible for charging is not addressed here because our understanding of the electronic properties of the metal-nanotube contacts is incomplete. ${ }^{19,20}$ We restrict the model to metallic nanotubes so that the conductivity along the axis is large compared to that in the perpendicular direction, where a contact barrier hinders the electronic conduction. We use the term "voltage" to describe the difference in the potential existing between the nanotube wall and the conducting fluid, which may be different from the potential applied externally in an experiment. Thus, the entire applied voltage is assumed to drop across the nanotube-mercury contact, creating an electric field in the space between the liquid and the wall.

According to the Lippmann model, ${ }^{18}$ the electrical double layer across the interface at the contact region corresponds to a capacitor with a certain electrostatic charge, $q$, and capacitance, $C$, associated with it. The free energy, $G$, of the contact with area $A$ is the sum of the energy of the neutral interface plus the electrostatic energy:

$$
G=A(\gamma-w)+q^{2} / 2 C
$$

Here, $\gamma$ is the surface tension of mercury, and $w>0$ is the work of adhesion per unit area between the mercury and the nanotube wall, expressed through the surface tension and static contact angle $\theta$ of the liquid droplet as follows:

$$
w=\gamma(1+\cos \theta)
$$

The wetting condition is for the change in free energy with area to be nonpositive

$$
\left(\frac{\partial G}{\partial A}\right)_{q} \equiv-\delta \leq 0
$$

where $-\delta$ is the interfacial tension, defined as free energy per unit area of the contact. The derivative in eq 5 is taken at $q=$ const, to differentiate the change in energy resulting from the variation of the geometrical shape of the interface from the change in energy produced by charge accumulation 
(driven by the power supply). Combining eqs $3-5$, one obtains for $\delta$

$$
\delta=\gamma \cos \theta+c V^{2} / 2
$$

where $V$ is the voltage applied to the interface and $c=C / A$ is the capacitance per unit area of the cylindrical surface. Thus, the wetting condition becomes

$$
c V^{2} / 2 \geq-\gamma \cos \theta
$$

For the liquid inside a cylindrical nanotube

$$
c=\frac{\epsilon_{0}}{r_{1} \ln \left(r_{2} / r_{1}\right)}
$$

where $r_{1}$ and $r_{2}$ are the radii of a liquid column and nanotube, and $\epsilon_{0}=8.85 \times 10^{-12} \mathrm{~F} / \mathrm{m}$, is an electric constant. Note that there is a difference in contact capacitance for mercury in the core versus that on the outer side of the nanotube walls.

In the simulations, electrowetting was modeled by considering the change, $\Delta E_{e}$, in the energy of the electrostatic field upon bringing a neutral atom to the charged interface from the bulk of the liquid. Because of electrostatic repulsion, the surface charge is redistributed over a greater area and the electrostatic energy is lowered by

$$
\Delta E_{e}=-c V^{2} / 2 \rho_{s}
$$

where $\rho_{s}=11.82 \mathrm{~nm}^{-2}$ is the surface density of mercury atoms. The energy depends on the geometry of the interface and will be slightly different for atoms located inside versus outside of the cylindrical nanotube. Exact treatment of the effect requires dynamic evaluation of atomic charges interacting through long-range Coulomb forces, which is computationally prohibitive. As an approximation for the actual interactions, a local external force acting along the normal was applied to each atom near the interface according to

$$
F_{\text {ext }}(r)= \begin{cases}F_{0}, & 0<r<a \\ 0, & r \geq a, r \leq 0\end{cases}
$$

where $r$ is the normal distance to the surface from the bulk of the liquid, $a=3.5 \AA$ is a characteristic distance, and $F_{0}$ $=\Delta E_{e} / a$. The direction of the force is toward the nanotube surface, and $a$ is chosen to include atoms from the second surface layer of the liquid. Although a simplification of the explicit electrostatic interactions, this model contains the salient features of electrowetting and should give a reasonable description of its dynamics. Equations 9 and 10 have been presented first in ref 12.

IV. Simulation of Electrowetting. The initial equilibrium configuration for simulating electrowetting and calculating imbibition speeds is shown in Figure 1a, where a 15.7-nmlong uncapped $(20,20)$ SWNT has been immersed in a bath of liquid consisting of 37013 mercury atoms. The size of
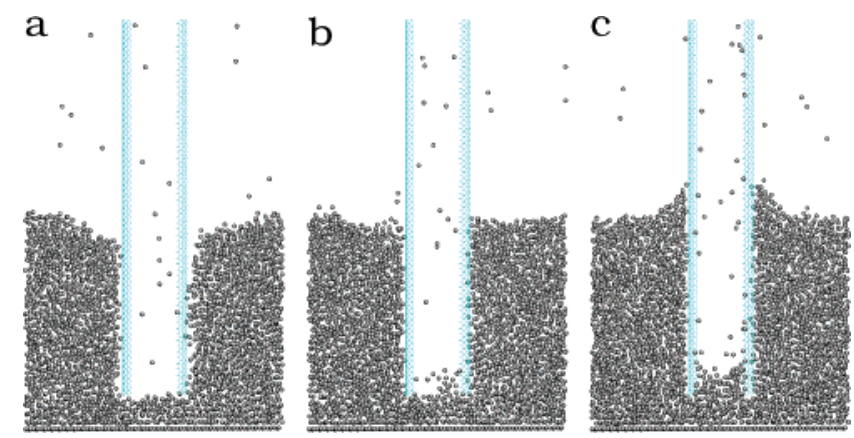

Figure 1. MD simulation results of the early stages of electrowetting in a $15.7-n m-l o n g ~(20,20)$ SWNT by mercury. (a) Snapshot at no applied bias $(t=0)$ showing a nonwetting meniscus at the outer walls of the nanotube submerged into mercury fluid; snapshots taken at (b) 20 ps and (c) 100 ps after the application of $3.5 \mathrm{~V}$ across the contact.
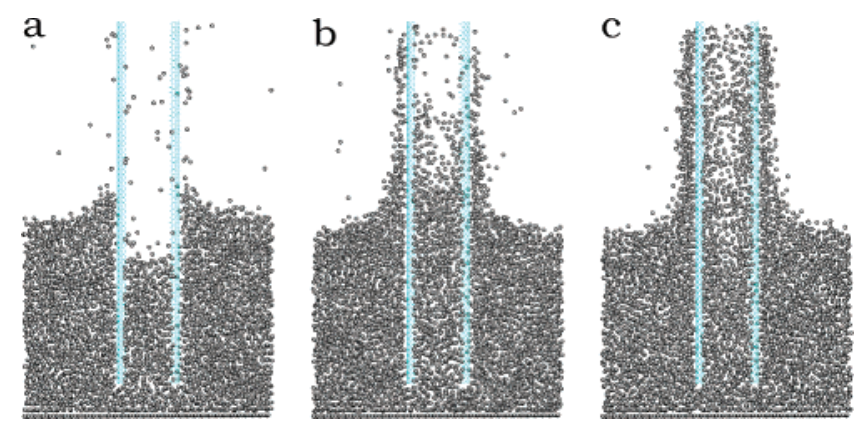

Figure 2. Snapshots of mercury electrowetting in a 15.7-nm-long $(20,20)$ SWNT taken 330 ps after the application of (a) $3.5 \mathrm{~V}$, (b) $4.0 \mathrm{~V}$, and (c) $4.5 \mathrm{~V}$ across the contact.

the simulation domain was $108 \times 104 \AA$ with periodic boundary conditions applied only in the lateral directions. The liquid was held in place by a single layer of fixed $\mathrm{Hg}$ atoms at the bottom of the bath. The rigid SWNT was dipped into the liquid at a constant speed of $10 \mathrm{~m} / \mathrm{s}$ until its open end reached a distance of $14 \AA$ from the bottom of the bath. Equilibration was achieved by coupling the system to a fixed temperature thermostat at $300 \mathrm{~K}$. A meniscus indicative of nonwetting behavior forms between the outer nanotube walls and the liquid. Consistent with the absence of wetting, mercury does not penetrate the core.

When the electrocapillary force is switched on, wetting of the SWNT by mercury takes place. Figures $1 \mathrm{~b}$ and $1 \mathrm{c}$ represent snapshots taken 20 and 100 ps after the application of $3.5 \mathrm{~V}$ across the contact, respectively. As mercury atoms begin to enter the nanotube, the nonwetting meniscus on the outer walls inverts and wetting is observed. Simultaneously, a column of mercury begins to form in the core, which moves as a single front at an initial speed of $\sim 13 \mathrm{~m} / \mathrm{s}$. The imbibition speed remains constant as the column front advances. Figure 2a illustrates the position $330 \mathrm{ps}$ after the application of $3.5 \mathrm{~V}$. Clearly absent is a wetting film along the nanotube wall advancing ahead of the column.

At larger electrowetting driving forces, obtained simply by increasing the applied voltage, a prewetting film appears to advance ahead of the column front. Figure 2 illustrates electrowetting in $(20,20)$ SWNTs as a function of voltage, $330 \mathrm{ps}$ after its application. It is obvious that wetting occurs 


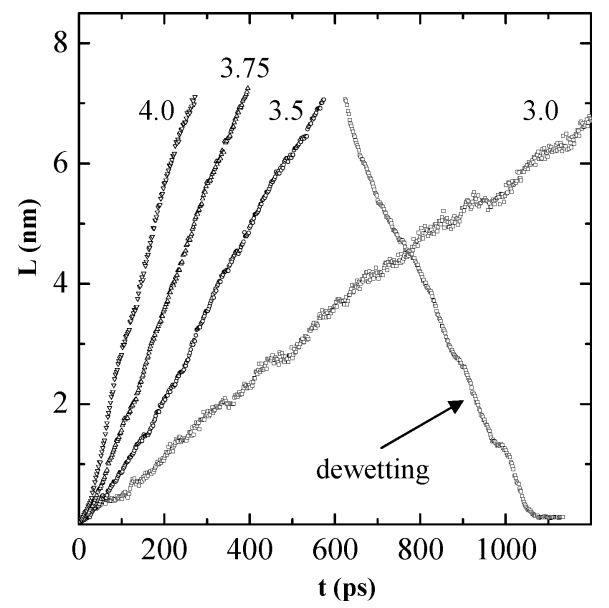

Figure 3. Core penetration length as a function of time in a 7.87nm-long $(20,20)$ SWNT at four different voltages (magnitude indicated in volts) applied across the contact. The length varies almost linearly with time during the initial wetting stage, permitting determination of imbibition velocities and mass transfer rates.

faster at higher voltages: the column is more advanced at $4.0 \mathrm{~V}$ (Figure 2b) as compared to $3.5 \mathrm{~V}$ (Figure 2a), while it appears to have filled the nanotube at $4.5 \mathrm{~V}$ (Figure 2c), although the latter will densify further with time. Given enough time, the entire inner core of the tube will be filled at any voltage over the threshold value for electrowetting ( $2.5 \mathrm{~V}$, see below). Formation of a continuous film on the outer walls appears to occur only at higher voltages. Electrowetting along the outer walls is less favorable (slower) than in the core of the nanotube because of curvature reversal and smaller capacitance.

To further understand the imbibition dynamics, we performed a series of computational runs at different contact potentials with shorter nanotubes $(L=7.87 \mathrm{~nm})$ without thermostat coupling. Check runs at constant temperature yielded identical results below $3 \mathrm{~V}$, whereas at higher voltages the imbibition was slower for a thermostat coupled liquid. The results for the shorter nanotubes were identical to those obtained for longer nanotubes $(L=15.7 \mathrm{~nm})$. Figure 3 shows the penetration length of the liquid column as a function of time inside a section of a $(20,20)$ SWNT with length $L=7.87 \mathrm{~nm}$ at five different applied voltages. The penetration length was obtained from the number of atoms in the nanotube at time $t$ normalized by the total number of atoms in a filled nanotube. The penetration length varies almost linearly with time during the initial filling process. Deviations from linearity are mainly due to fluctuations resulting from the small system size.

Because the penetration length is linear in time, constant imbibition rates (atom flux) can be obtained and plotted versus the applied voltage, as shown in Figure 4. The imbibition rate can be approximated by a parabolic function of voltage and, thus, it follows the same quadratic scaling as the wetting force (see eq 6). This dependence suggests a linear relationship between the initial imbibition rate and the wetting force. A well-defined liquid column is present in the nanotube core below $3.75 \mathrm{~V}$, rendering possible the precise determination of the imbibition speed. Above 3.75

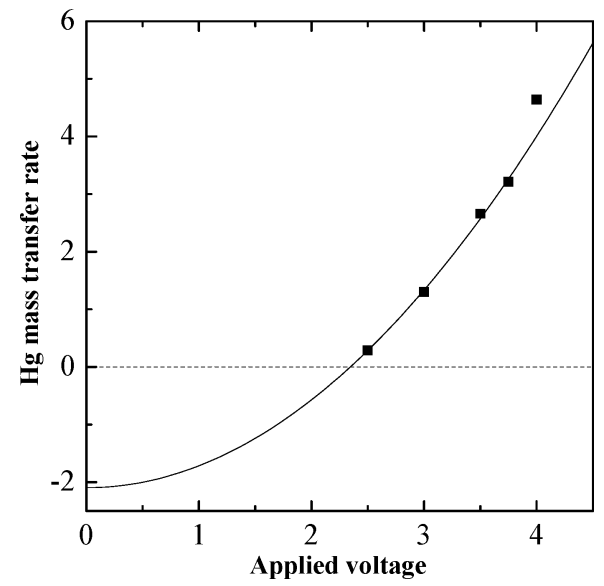

Figure 4. Imbibition rate of mercury atoms in the core of a $(20,20)$ SWNT as a function of applied voltage. The curve represents a quadratic fit to the points.

$\mathrm{V}$, appearance of the pre-wetting film makes estimation of the imbibition rate less precise. When a potential is no longer applied, the liquid column drains rapidly at a uniform speed of $\sim 15 \mathrm{~m} / \mathrm{s}$, as shown in Figure 4 by the descending line. The system returns to its initial state, from which a new round of the reversible filling process can be initiated.

Unlike core filling, spreading of mercury along the outer walls does not proceed at a constant rate. Initially, mercury atoms spread rapidly to cover the outer walls. Then, the wetting film thickness increases more gradually. This behavior reveals the importance of electrical continuity in the spreading film, and identifies a possible weakness in the employed model. Electrical continuity is essential for calculating the capacitance between the wetting film and the nanotube and, subsequently, the magnitude of the electrowetting driving force. If the spreading film is discontinuous or very thin (i.e., a couple of monolayers), then it may not support electrical conduction, thus limiting electrowetting. We therefore refrain from discussing wetting speeds and atom transport rates for the outer walls. The presence of a column of liquid in the core, especially when it advances as a single front, suggests that the electrical continuity of mercury inside the nanotube is not impaired and, thus, the imbibition rate calculations are not subject to this uncertainty.

Another important quantity characterizing capillary wetting is the hydrostatic force exerted by the fluid inside the capillary on an impenetrable barrier in its path. The electrocapillary pressure (ECP) associated with this force can be calculated by placing a repulsive wall perpendicular to the nanotube axis and measuring an average force of interaction with the liquid inside the nanotube at constant temperature. The radius of the liquid in a $(20,20)$ SWNT was assumed to be $10.56 \AA$, and the forces were averaged over 150 ps. As expected from eq 8, the ECP exhibits a quadratic dependence on applied bias (see Figure 5). The threshold for electrowetting can thus be calculated as the voltage where the ECP is equal to zero. For a $(20,20)$ SWNT, the threshold voltage is $2.5 \mathrm{~V}$. The ECP increases quickly with voltage to reach $2.4 \mathrm{kbar}$ at $4.0 \mathrm{~V}$, indicating that substantial pressures can be obtained at relatively small applied voltages. However, 


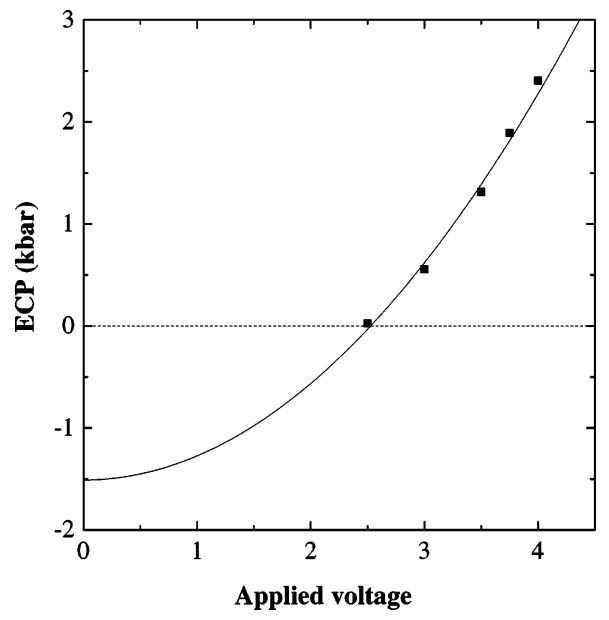

Figure 5. Electrocapillary pressure of mercury inside a $(20,20)$ SWNT as a function of applied voltage obtained from MD simulations. The curve represents a quadratic fit to the points.

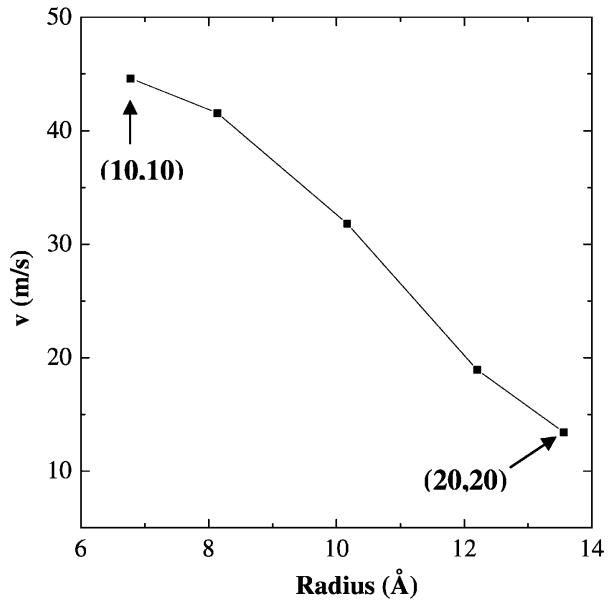

Figure 6. Imbibition speeds of mercury in the core of $(10,10)$, $(12,12),(15,15),(18,18)$, and $(20,20)$ SWNTs at a bias voltage of $3.5 \mathrm{~V}$.

no crystallization is expected for mercury in the inner core of the nanotube until the pressure reaches a value close to $\sim 12 \mathrm{kbar}$, which is the experimental value for bulk solidification at room temperature. ${ }^{21}$ The voltage required to reach this pressure is $\sim 9 \mathrm{~V}$, which far exceeds the threshold voltage for shortening the nanotube during pulsed etching. ${ }^{12}$

The imbibition speed also depends on the size of the nanotube. In Figure 6, we plot mercury imbibition speeds in armchair SWNTs of various diameters with chiral vectors between $(20,20)$ and $(10,10)$ at a constant applied voltage of $3.5 \mathrm{~V}$. The speed increases almost linearly with diminishing nanotube size as a result of an increase in the capacitance per unit area (curvature effect, see eq 10). The linear time dependence of the penetration length is preserved in all nanotubes down to the $(10,10)$ SWNT. Loss of the metallic properties of mercury in the core may limit the applicability of the electrowetting model to very small nanotubes.

V. Discussion. Although the flow mechanics in capillaries of micrometer size can still be described by continuum methods, ${ }^{22,23}$ the validity of this approximation for flow in carbon nanotubes must be scrutinized. When gravity effects are negligible, filling of macroscopic capillaries via laminar flow by a wetting fluid has been described by the LucasWashburn equation ${ }^{22}$

$$
\rho\left[l \frac{d^{2} l}{d t^{2}}+\left(\frac{d l}{d t}\right)^{2}\right]+\frac{8 \eta l}{r^{2}} \frac{d l}{d t}=\frac{2 \delta}{r}
$$

where: $l(t)$ is the filled column length as a function of time $t, \rho$ and $\eta$ are the fluid density and viscosity, $r$ is the radius of the column, and $-\delta$ is the interfacial tension between the fluid and the wall of the capillary. The two terms on the left-hand side of eq 11 relate to the inertial and viscous resistance of the imbibing fluid, respectively, and the term on the right-hand side is the capillary driving force. The analytical solution to the Lucas-Washburn equation can be expressed as

$$
l(t)=\left(\frac{r \delta}{2 \eta}\right)^{1 / 2}\left(t+\frac{1}{a}(\exp (-\beta t)-1)\right)^{1 / 2}
$$

where $\beta=8 \eta / r^{2} \rho$. At long times, eq 12 predicts that the fluid will imbibe the capillary column with a square-root dependence on time. At the initial stages of imbibition, when $\beta t \ll 1$, eq 12 can be approximated by a linear dependence

$$
l(t)=\left(\frac{2 \delta}{\rho r}\right)^{1 / 2} t
$$

This approximation has been used by Supple and Quirke ${ }^{24}$ to explain the linear time-dependence of the number of oil (decane) molecules imbibing into the core of carbon nanotubes of various sizes. Equation 13 could similarly be used in our case to explain the calculated linear time dependence for the rate of mercury atoms imbibing a SWNT, as shown in Figure 3. However, eq 13 predicts an imbibition speed that depends linearly on voltage, which is in disagreement with the quadratic dependence demonstrated in Figure 4. Furthermore, assuming $r \approx 1.1 \mathrm{~nm}$ for a $(20,20)$ SWNT, $\rho \approx 13.6 \mathrm{~g} / \mathrm{cm}^{3},{ }^{25}$ and $\eta \approx 1.5 \times 10^{-3} \mathrm{Ns} / \mathrm{m}^{2}$ for $\mathrm{Hg}$ at $300 \mathrm{~K},{ }^{26}$ one obtains $\beta \approx 8 \times 10^{11} \mathrm{~s}^{-1}$, which limits the range of validity of eq 13 to $t \ll 1.26 \mathrm{ps}$. This range is considerably smaller than that seen in the MD simulations, where the linear time-dependence extends to hundreds of picoseconds.

The above analysis suggests that the Lucas-Washburn equation in the form eq 11 fails to explain two key dependencies in the mercury imbibition of nanotubes. The deficiency may be related to the capillary driving force responsible for imbibition. For a fixed voltage above the threshold value for electrowetting, the capillary driving force depends on contact angle (see eq 6). This angle is associated with a moving wetting line and may be influenced by the wetting-line velocity as proposed by Blake et al. ${ }^{27}$ To better explain simulated speeds of capillary penetration ${ }^{28}$ and droplet spreading, ${ }^{29}$ Blake et al. introduced a modification to the Lucas-Washburn equation to account for a velocitydependent dynamic contact angle, which reduces the capillary 
driving force at the onset of imbibition. Their hypothesis translates into a functional dependence of the dynamic wetting force, $\delta^{\prime}$, on the speed of the wetting line, as follows

$$
\delta^{\prime}=\delta-\xi \frac{d l}{d t}
$$

where $\zeta$ is a "friction" coefficient and $\delta$ is the wetting force at a wetting-line velocity of zero. Possible physical reasons for this "wetting-line friction" can be found in ref 27. Taking the radius, $r$, of the fluid column as a small parameter, substitution of eq 14 into eq 11 yields

$$
\frac{4 \eta l}{r} \frac{d l}{d t}+\zeta \frac{d l}{d t}-\delta=0
$$

which can be solved analytically to obtain

$$
l(t)=\frac{\xi r}{4 \eta}(\sqrt{1+b t}-1)
$$

where $b=8 \eta \delta / r \zeta^{2}$. Again, at the early stages of imbibition when $b t \ll 1$, eq 16 can be simplified

$$
l(t)=\frac{\delta}{\zeta} t
$$

Equation 17 predicts the correct scaling on $\delta$ and, thus, captures the quadratic dependence of the imbibition rate on applied voltage. The friction constant, $\zeta$, may be obtained from eq 17 using the calculated imbibition velocities and values of the interfacial tension at different voltages. For the four voltages from the interval $2.75-4.0 \mathrm{~V}$ above the electrowetting threshold, one obtains $\zeta=(6.96 \pm 0.63) \times$ $10^{-3} \mathrm{~kg} / \mathrm{m} \cdot \mathrm{s}$, which extends the range of the linear timedependency to $t \ll 42.4$ ps at $V=3.5 \mathrm{~V}$. This value is in better agreement with the results of the simulation, although it still underestimates the linearity region, possibly because of the use of the bulk viscosity of mercury.

The concept of "wetting-line friction" helps explain two key dependencies of the imbibition of carbon nanotubes by mercury and, thus, it extends the range of validity of the Lucas-Washburn equation to capillarity at the nanoscale where imbibition speeds are substantial and viscous forces are relatively small. Similar modifications to macroscopic equations may capture other phenomena in the emerging field of nanofluidics.

VI. Summary and Conclusions. The dynamics of electrowetting of carbon nanotubes by mercury were studied by molecular dynamics simulations in conjunction with a simple model of electrocapillarity. A scaled ab initio $\mathrm{Hg}_{2}$ potential was used to describe liquid mercury, while the mercurycarbon interaction was modeled by a Lennard-Jones potential optimized to reproduce the experimental contact angle of a mercury drop on an oriented graphite surface. We found that electrocapillarity can produce a large driving force for wetting the inner core of open carbon nanotubes. The length of the liquid mercury column propagating in the core was characterized by a linear dependence on time. Both electrocapillary pressure and imbibition speed displayed a quadratic dependence on applied voltage. The rapid speed of draining of mercury from the nanotube core upon termination of electrowetting (i.e., at zero potential) suggests that very fast cycling between filled-empty states is possible. The imbibition velocity decreased with nanotube size. A macroscopic wetting model based on the Lucas - Washburn equation was capable of capturing the observed scalings by introducing a wetting-line friction term to account for a velocity-dependent contact angle.

Acknowledgment. We thank Prof. C. P. Collier for insightful discussions and a critical reading of the manuscript. This theoretical work was supported in part by NSF (grants CTS-0404353 and CTS-0508096).

\section{References}

(1) Pederson, M. R.; Broughton, J. Q. Phys. Rev. Lett. 1992, 69, 2689.

(2) Ajayan, P. M.; Iijima, S. Nature 1993, 361, 333.

(3) Guerret-Plecourt, C.; Le Bouar, Y.; Loiseau, A.; Pascard, H. Nature 1994, 372, 761.

(4) Kiang, C. H.; Choi, J. S.; Tran, T. T.; Bacher, A. D. J. Phys. Chem. B 1999, 103, 7449 .

(5) Tsang, S. C.; Chen, Y. K.; Harris, P. J. F.; Green, M. L. H. Nature 1994, 372, 159.

(6) Ebbesen, T. W. J. Phys. Chem. Solids 1996, 57, 951.

(7) Ajayan, P. M.; Ebbesen, T. W. Rep. Prog. Phys. 1997, 60, 1025

(8) Monthioux, M. Carbon 2002, 40, 1809.

(9) Dujardin, E.; Ebbesen, T. W.; Hiura, H.; Tanigaki, K. Science 1994, $265,1850$.

(10) Adam, N. K. The Physics and Chemistry of Surfaces; Dover: New York, 1968.

(11) Grahame, D. C. Chem. Rev. 1947, 41, 441.

(12) Chen, J. Y.; Kutana, A.; Collier, C. P.; Giapis, K. P. Science 2005, 310, 1480 .

(13) Paulus, B.; Rosciszewski, K. Chem. Phys. Lett. 2004, 394, 96.

(14) Rademann, K.; Kaiser, B.; Even, U.; Hensel, F. Phys. Rev. Lett. 1987, 59, 2319.

(15) Supplemental online material for ref 12, accessible through: www.sciencemag.org.

(16) Schwerdtfeger, P.; Wesendrup, R.; Moyano, G. E. J. Chem. Phys. 2001, 115, 7401.

(17) Awasthi, A.; Bhatt, Y. J.; Garg, S. P. Meas. Sci. Technol. 1996, 7, 753.

(18) Lippmann, G. Ann. Chim. Phys. 1875, 5, 494.

(19) (a) Heinze, S.; Tersoff, J.; Martel, R.; Derycke, V.; Appenzeller, J.; Avouris, Ph. Phys. Rev. Lett. 2002, 89, 106801. (b) Javey, A.; Guo, J.; Wang, Q.; Lundstrom, M.; Dai, H. Nature 2003, 424, 654.

(20) Shan, B.; Cho, K. Phys. Rev. B 2004, 70, 233405.

(21) Klement, W., Jr.; Jayaraman, A.; Kennedy, G. C. Phys. Rev. 1963 131,1 .

(22) (a) Lucas, R. Kolloid-Z. 1918, 23, 15. (b) Washburn, E. W. Phys. Rev. 1921,17, 273.

(23) Tas, N. R.; Haneveld, J.; Jansen, H. V.; Elwenspoek, M.; van den Berg, A. Appl. Phys. Lett. 2004, 85, 3274.

(24) (a) Supple, S.; Quirke, N. J. Chem. Phys. 2004, 121, 8571; (b) Supple, S.; Quirke, N. J. Chem. Phys. 2005, 122, 104706.

(25) Ambrose, D. Metrologia 1990, 27, 245.

(26) Grosse, A. V. J. Phys. Chem. 1964, 68, 3419.

(27) Blake, T. D.; Haynes, J. M. J. Colloid Interface Sci. 1969, 30, 421.

(28) Martic, G.; Gentner, F.; Seveno, D.; Coulon, D.; De Coninck, J.; Blake, T. D. Langmuir 2002, 18, 7971.

(29) de Ruijter, M. J.; Blake, T. D.; De Coninck, J. Langmuir 1999, 15, 7836

NL052393B 